\title{
A method to simplify perturbation analyses of periodical flows
}

Proc IMechE Part M:

J Engineering for the Maritime Environment 2016, Vol. 230(I) 22-32

(C) IMechE 2014

Reprints and permissions:

sagepub.co.uk/journalsPermissions.nav DOI: 10.1 I77//475090214529425

pim.sagepub.com

(SSAGE

\section{Heinrich Söding}

\begin{abstract}
Perturbation methods up to first order with respect to motion or wave amplitude are common in seakeeping predictions. For higher than first order, lengthy theoretical analyses are required. They result in complicated formulae requiring high programming effort, and often the well-established numerical methods for first-order quantities fail when applied to second-order flow quantities. Both the derivation of the required expressions and their programming are simplified by using mathematical entities called perturbators. The concept of perturbators is described, and their application is demonstrated, for a two-dimensional test problem: A cylinder with horizontal axis partly immersed into an ideal fluid. The cylinder performs sinusoidal heave, sway, and roll motions. Stationary and double-frequency second-order forces are determined. For a heaving semicircle, vertical forces are determined numerically and compared to published results.
\end{abstract}

\section{Keywords}

Potential flow, free surface, oscillatory motion, heave, sway, flow force

Date received: 21 January 2014; accepted: 24 February 2014

\section{Introduction}

Stokes waves constitute probably the best-known periodical flow analyzed by perturbation analysis. However, many other time periodical flows are subject to perturbation analyses also today because fully nonlinear simulations often need too much computer time. That is usually the case if the statistical properties of long-time motions of and loads on bodies such as ships, floating, or ground-based marine structures must be determined in natural, time-variable seaways. In such cases, the basis for computing load and motion statistics are transfer functions, that is, responses to regular waves of different frequencies and directions. If besides first-order responses (i.e. those proportional to wave amplitude) also second-order responses are of interest, the basis for predictions is responses to pairs of regular waves differing usually (but not always) by frequency and direction. Examples where second- or higher order analyses are required are the added resistance of ships in a seaway (a pure second-order phenomenon), motions of moored structures (here second-order difference-frequency responses are dominant), and vibrations of ships ("springing") and marine structures, especially tension-leg platforms, where second-order sum-frequency responses are important.

A difficulty with perturbation analyses of higher than first order is that they, usually, result in complicated formulae. Both their derivation and programming are cumbersome and error-prone. This article shows a method to simplify both tasks by introducing a new mathematical entity called perturbator. In the following, the concept of perturbators is explained, and their use is demonstrated, using as example the twodimensional (2D) second-order free-surface flow around a periodically moving body in a regular wave. The results for heaving of a semicircle are compared to other experimental and computational work.

\section{Perturbators of second order for one frequency}

\section{Concept}

Consider a real, scalar quantity $a(t)$ oscillating harmonically over time $t$ with circular frequency $\omega$

$$
a(t)=a_{0}+\operatorname{Re}\left(a_{1} e^{i \omega t}\right)
$$

Technical University of Hamburg-Harburg, Institute for Fluid Dynamics and Ship Theory, Hamburg, Germany

\section{Corresponding author:}

Heinrich Söding, Technical University of Hamburg-Harburg, Institute for Fluid Dynamics and Ship Theory, Schwarzenbergstr. 95 C, 21073

Hamburg, Germany.

Email: h.soeding@tu-harburg.de 
where $a_{0}$ is a real constant and $a_{1}$ is a complex constant called complex amplitude of $a . a_{0}$ and $a_{1}$ are considered as quantities of zeroth and first order, respectively.

A corresponding equation is assumed to hold for another quantity $b$

$$
b(t)=b_{0}+\operatorname{Re}\left(b_{1} e^{i \omega t}\right)
$$

$a$ and $b$ are supposed to oscillate with the same frequency $\omega$. (The case of different $\omega$ values is considered later.)

The product $a b$ depends on time as follows

$$
\begin{aligned}
a(t) b(t)= & {\left[a_{0}+\operatorname{Re}\left(a_{1} e^{i \omega t}\right)\right]\left[b_{0}+\operatorname{Re}\left(b_{1} e^{i \omega t}\right)\right] } \\
= & a_{0} b_{0}+a_{0} \operatorname{Re}\left(b_{1} e^{i \omega t}\right)+\operatorname{Re}\left(a_{1} e^{i \omega t}\right) b_{0} \\
& +\operatorname{Re}\left(a_{1} e^{i \omega t}\right) \operatorname{Re}\left(b_{1} e^{i \omega t}\right)
\end{aligned}
$$

Using the formula (correct for arbitrary complex numbers $z$ )

$$
\operatorname{Re}(z)=\frac{1}{2}\left(z+z^{*}\right)
$$

where the asterisk indicates the complex conjugate, the last term in equation (4) can be transformed to

$$
\begin{aligned}
& \operatorname{Re}\left(a_{1} e^{i \omega t}\right) \operatorname{Re}\left(b_{1} e^{i \omega t}\right)=\operatorname{Re}\left(a_{1} e^{i \omega t} \frac{1}{2}\left(b_{1} e^{i \omega t}+b_{1}^{*} e^{-i \omega t}\right)\right) \\
& =\frac{1}{2} \operatorname{Re}\left(a_{1} b_{1} e^{2 i \omega t}\right)+\frac{1}{2} \operatorname{Re}\left(a_{1} b_{1}^{*}\right)
\end{aligned}
$$

Thus, from equation (4) follows

$$
\begin{aligned}
a(t) b(t)= & \underbrace{a_{0} b_{0}}_{c_{0}}+\operatorname{Re}[\underbrace{\left(a_{0} b_{1}+a_{1} b_{0}\right)}_{c_{1}} e^{i \omega t}] \\
& +\underbrace{\frac{1}{2} \operatorname{Re}\left(a_{1} b_{1}^{*}\right)}_{c_{2}}+\operatorname{Re}(\underbrace{\frac{1}{2} a_{1} b_{1}}_{c 3} e^{2 i \omega t})
\end{aligned}
$$

The right-hand side of equation (8) is interpreted as the general form of quantities appearing in secondorder perturbation analyses

$$
c(t)=c_{0}+\operatorname{Re}\left(c_{1} e^{i \omega t}\right)+c_{2}+\operatorname{Re}\left(c_{3} e^{2 i \omega t}\right)
$$

Here, $c_{0}$ is of order $0, c_{1}$ is of order 1 , and $c_{2}$ and $c_{3}$ are both of order $2 ; c_{0}$ and $c_{2}$ are real, and $c_{1}$ and $c_{3}$ are complex. One could combine the two constants $c_{0}$ and $c_{2}$ to a single quantity; however, this is not done here because of the different order of both quantities.

For fixed $\omega$, the first-order time function $a$ in equation (1) is uniquely defined (represented) by $a_{0}$ and $a_{1}$. Correspondingly, the second-order quantity $c$ is represented by $c_{0}-c_{3}$. The ordered set of these four quantities is called a perturbator of second order for one frequency. In the following, perturbators will be indicated by boldface symbols or, if their elements $c_{0}-c_{3}$ are indicated separately, by brackets $\langle$ and $\rangle$

$$
\mathbf{c}=\left\langle c_{0}, c_{1}, c_{2}, c_{3}\right\rangle
$$

Analyses involving only harmonically oscillating functions are simplified by dealing not directly with the time functions, but with their complex amplitudes. The same is not possible in second-order analyses because they involve, additionally, terms oscillating with frequency $2 \omega$, and also constant terms that interact with the oscillating terms. However, in this case, the use of perturbators simplifies the analyses even more than complex amplitudes in first-order analyses. Programming of numerical evaluations is simplified correspondingly if perturbators are introduced as new types of variables and if their mathematical operations are programmed.

Even more pronounced simplifications are found in third-order analyses using perturbators of third order (see later).

\section{Computing with scalar second-order one-frequency perturbators}

To apply perturbators in mathematical expressions, we need to define rules for addition and multiplication. Addition is defined such that the sum of two secondorder time functions $a(t)+b(t)$ is represented by the sum of perturbators $\mathbf{a}+\mathbf{b}$, and correspondingly, the product of time functions is represented by the product of perturbators ab.

The sum of two second-order time functions $a(t)$ and $b(t)$ is

$$
\begin{gathered}
c(t)=a(t)+b(t)=a_{0}+b_{0}+\operatorname{Re}\left(\left(a_{1}+b_{1}\right) e^{i \omega t}\right) \\
+a_{2}+b_{2}+\operatorname{Re}\left(\left(a_{3}+b_{3}\right) e^{2 i \omega t}\right)
\end{gathered}
$$

It is represented by the perturbator

$$
\mathbf{c}=\mathbf{a}+\mathbf{b}=\left\langle a_{0}+b_{0}, a_{1}+b_{1}, a_{2}+b_{2}, a_{3}+b_{3}\right\rangle
$$

Thus, the sum of perturbators is defined equivalently to the vector sum: corresponding elements are added.

The product of two second-order time functions is

$$
\begin{gathered}
c(t)=a(t) b(t)=\left[a_{0}+\operatorname{Re}\left(a_{1} e^{i \omega t}\right)+a_{2}+\operatorname{Re}\left(a_{3} e^{2 i \omega t}\right)\right] \\
{\left[b_{0}+\operatorname{Re}\left(b_{1} e^{i \omega t}\right)+b_{2}+\operatorname{Re}\left(b_{3} e^{2 i \omega t}\right)\right]} \\
\approx a_{0} b_{0}+\operatorname{Re}\left[\left(a_{0} b_{1}+a_{1} b_{0}\right) e^{i \omega t}\right]+a_{0} b_{2}+a_{2} b_{0} \\
+\frac{1}{2} \operatorname{Re}\left(a_{1} b_{1}^{*}\right)+\operatorname{Re}\left[\left(a_{0} b_{3}+a_{3} b_{0}+\frac{1}{2} a_{1} b_{1}\right) e^{2 i \omega t}\right]
\end{gathered}
$$

The symbol $\approx$ means equality up to second order; terms of higher than second order are omitted, as typical in perturbation analyses.

The time function in equation (14) is represented by the perturbator

$$
\begin{aligned}
& \mathbf{c}=\mathbf{a b}==\langle\underbrace{a_{0} b_{0}}_{c_{0}}, \underbrace{a_{0} b_{1}+a_{1} b_{0}}_{c_{1}}, \\
& \underbrace{a_{0} b_{2}+a_{2} b_{0}+\frac{1}{2} \operatorname{Re}\left(a_{1} b_{1}^{*}\right)}_{c_{2}}, \underbrace{a_{0} b_{3}+a_{3} b_{0}+\frac{1}{2} a_{1} b_{1}}_{c_{3}}\rangle
\end{aligned}
$$


Equation (15) is the rule for multiplying perturbators.

The rules satisfied by this definition of addition and multiplication of scalar perturbators are now examined:

- There exists a zero element for addition which does not change the sum: $\mathbf{c}=\langle 0,0,0,0\rangle$.

- There exists a one element for multiplication which does not change the product: $\mathbf{c}=\langle 1,0,0,0\rangle$.

- The inverse of addition (subtraction) exists: $\mathbf{a}-\mathbf{b}=\left\langle a_{0}-b_{0}, a_{1}-b_{1}, a_{2}-b_{2}, a_{3}-b_{3}\right\rangle$.

- The inverse of multiplication (division) exists if the element $b_{0}$ of the divisor is $\neq 0$. It is defined as $\mathbf{a} / \mathbf{b}=\mathbf{a b}^{-1}$ with

$$
\mathbf{b}^{-1}=\left\langle\frac{1}{b_{0}}, \quad \frac{-b_{1}}{b_{0}^{2}}, \quad \frac{-b_{2}}{b_{0}^{2}}+\frac{\left|b_{1}\right|^{2}}{2 b_{0}^{3}}, \quad \frac{-b_{3}}{b_{0}^{2}}+\frac{b_{1}^{2}}{2 b_{0}^{3}}\right\rangle
$$

- Addition and multiplication are associative and commutative.

- The distributive law is satisfied.

Thus, one can compute with perturbators as with real scalars, with a minor exception regarding division through quantities like $\langle 0,1,0,0\rangle$ which are $\neq \mathbf{0}$, but for which the zero-order element is 0 . (In the following applications, division of perturbators does not occur.)

\section{Vector and matrix perturbators}

The definition of perturbators is now extended by accepting as elements not only scalars but also vectors and matrices: real vectors and matrices for the elements $c_{0}$ and $c_{2}$, and complex ones for the elements $c_{1}$ and $c_{3}$. The definition of the addition remains unchanged, while for multiplication the symbol " $*$ " is written here. It stands for all relevant kinds of multiplication: the scalar product, the vector product of three-dimensional (3D) vectors, the matrix product for matrices, and the product of a scalar with a vector or a matrix. Thus, the generalized multiplication rule for second-order onefrequency perturbators is

$$
\begin{aligned}
& \mathbf{c}=\mathbf{a} * \mathbf{b}=\langle\underbrace{a_{0} * b_{0}}_{c_{0}}, \underbrace{a_{0} * b_{1}+a_{1} * b_{0}}_{c_{1}}, \\
& \underbrace{a_{0} * b_{2}+a_{2} * b_{0}+\frac{1}{2} \operatorname{Re}\left(a_{1} * b_{1}^{*}\right)}_{c_{2}}, \\
& \underbrace{a_{0} * b_{3}+a_{3} * b_{0}+\frac{1}{2} a_{1} * b_{1}}_{c_{3}}\rangle
\end{aligned}
$$

As before, it is easily shown that addition and multiplication of perturbators correspond to the respective operations with the time functions they represent, may they be real scalars, vectors, or matrices.
However, of the computing rules listed above for scalar perturbators, only those hold in this more general case, which are satisfied also for the respective product "*." For example, there is no inverse of the vector product; the commutative law does not hold (in general) for vector and matrix products, and the associative law does not hold for the scalar product of vectors and for the vector product.

\section{Further operations}

For dealing with perturbators, it is helpful to define a few more operations: addition and multiplication of a perturbator b with a real, time-independent (constant) quantity $a$ of scalar, vector, or matrix type is admitted. The sum or product is defined to be the same as if $a$ were extended to the perturbator $\langle a, 0,0,0\rangle$ before the addition or multiplication was performed. That means, in case of addition, the zero-order element of $\mathbf{b}$ is increased by $a$, whereas in multiplication all elements of $\mathbf{b}$ are multiplied by $a$.

The above restriction to real constant quantities $a$ is important. It is necessary to avoid that the element $c_{0}$ of the resulting perturbator becomes complex. However, multiplication with complex constants occurs frequently in first-order perturbation analyses, for example, when computing a response amplitude as product of the complex wave amplitude (first-order perturbator representing the wave motion) with the complex transfer function (a constant). Here, multiplication with a complex factor means that amplitude and phase of the original time function $b(t)$ are changed: multiplication with the complex constant

$$
a=a_{r}+i a_{i}
$$

corresponds to applying the operator

$$
a_{r}+\frac{a_{i}}{\omega} \frac{\partial}{\partial t}
$$

to the time function $b(t)$. As the same operation occurs also in second-order perturbation analyses, the multiplication of perturbators with complex constants is defined such that it corresponds to applying operator (19) to the time function represented by the perturbator. This results in the following multiplication rule

$$
\begin{aligned}
& \underbrace{\left(a_{r}+i a_{i}\right)}_{a}\left\langle b_{0}, b_{1}, b_{2}, b_{3}\right\rangle \\
& =\left\langle a_{r} b_{0},\left(a_{r}+i a_{i}\right) b_{1}, a_{r} b_{2},\left(a_{r}+2 i a_{i}\right) b_{3}\right\rangle
\end{aligned}
$$

This definition of the product of a complex constant $a$ with a perturbator $b$ does not correspond to the perturbator multiplication of $b$ with $\langle a, 0,0,0\rangle$, nor with $\langle 0, a, 0,0\rangle$. The first alternative is impossible because it requires a real constant $a$, and the second would correspond to multiplication with an oscillating, not a constant quantity.

Finally, it is advantageous to define for perturbators the equivalent of the partial time derivative of the 
function represented by the perturbator. (The perturbator itself is constant over time.) Here, the equivalent operation is symbolized by a dot above the perturbator or behind a perturbator-valued expression. From equation (9) follows

$$
\dot{\mathbf{a}}=\left\langle a_{0}, a_{1}, a_{2}, a_{3}\right\rangle^{\bullet}=\left\langle 0, i \omega a_{1}, 0,2 i \omega a_{3}\right\rangle
$$

\section{Perturbators of different order for one frequency}

If one is interested only in first-order time-variable and second-order stationary quantities, instead of equation (10) one should use perturbators defined by only three quantities

$$
\mathbf{a}=\left\langle a_{0}, a_{1}, a_{2}\right\rangle
$$

If there are no quantities of order 0 , the first element $a_{0}$ of all perturbators can be eliminated. If there exist zero-order quantities, but are of no interest, one has to retain nonetheless the elements $a_{0}$ because they have influence on other elements in products of perturbators. Generally, perturbators can be adapted to different problems by restricting their length to the highest element which is of interest.

Perturbation analyses up to third order for only one frequency give time functions of the form

$$
\begin{aligned}
a(t)= & a_{0}+\operatorname{Re}\left(a_{1} e^{i \omega t}\right)+a_{2}+\operatorname{Re}\left(a_{3} e^{2 i \omega t}\right) \\
& +\operatorname{Re}\left(a_{4} e^{i \omega t}\right)+\operatorname{Re}\left(a_{5} e^{3 i \omega t}\right)
\end{aligned}
$$

The last two terms are of third order. $a(t)$ is represented by the third-order perturbator

$$
\mathbf{a}=\left\langle a_{0}, a_{1}, a_{2}, a_{3}, a_{4}, a_{5}\right\rangle
$$

Addition of third-order perturbators is defined as addition of the corresponding elements of both summands. The formula for the general product $*$ is, as before, derived from the rule that the perturbator product represents the product of the two third-order time functions. This results in

$$
\begin{aligned}
& \mathbf{c}=\mathbf{a} * \mathbf{b}=\langle\underbrace{a_{0} * b_{0}}_{c_{0}}, \underbrace{a_{0} * b_{1}+a_{1} * b_{0}}_{c_{1}}, \\
& \underbrace{a_{0} * b_{2}+a_{2} * b_{0}+\frac{1}{2} \operatorname{Re}\left(a_{1} * b_{1}^{*}\right)}_{c_{2}}, \\
& \underbrace{a_{0} * b_{3}+a_{3} * b_{0}+\frac{1}{2} a_{1} * b_{1}}_{c_{3}}, \\
& \underbrace{a_{0} * b_{4}+a_{4} * b_{0}+a_{1} * b_{2}+a_{2} * b_{1}+\frac{1}{2} a_{1}^{*} * b_{3}+\frac{1}{2} a_{3} * b_{1}^{*}}_{c_{4}}, \\
& \underbrace{a_{0} * b_{5}+a_{5} * b_{0}+\frac{1}{2} a_{1} * b_{3}+\frac{1}{2} a_{3} * b_{1}}_{c_{5}}\rangle
\end{aligned}
$$

\section{Second-order perturbators for two frequencies}

The above-described perturbators represent timeperiodic functions which occur due to interactions of time-harmonic functions if both contributors oscillate with the same frequency (or are time constant). Here, on the other hand, perturbators are introduced which describe the interaction of two time-harmonic functions oscillating with different frequencies

$$
a(t)=a_{0}+\operatorname{Re}\left(a_{1} e^{i \omega_{a} t}\right) ; \quad b(t)=b_{0}+\operatorname{Re}\left(b_{1} e^{i \omega_{b} t}\right)
$$

The sum, that is, the superposition of the two functions, consists of a constant term $a_{0}+b_{0}$ and two terms oscillating harmonically with frequencies $\omega_{a}$ and $\omega_{b}$. For perturbation analyses of higher order, the product $a(t) b(t)$ is of main interest

$$
\begin{aligned}
a(t) b(t) & =a_{0} b_{0}+\operatorname{Re}\left(b_{0} a_{1} e^{i \omega_{a} t}\right)+\operatorname{Re}\left(a_{0} b_{1} e^{i \omega_{b} t}\right) \\
& +\operatorname{Re}\left(a_{1} e^{i \omega_{a} t} \frac{1}{2}\left(b_{1} e^{i \omega_{b} t}+b_{1}^{*} e^{-i \omega_{b} t}\right)\right) \\
=a_{0} b_{0} & +\operatorname{Re}\left(b_{0} a_{1} e^{i \omega_{a} t}\right)+\operatorname{Re}\left(a_{0} b_{1} e^{i \omega_{b} t}\right) \\
+ & \operatorname{Re}\left(\frac{1}{2} a_{1} b_{1}^{*} e^{i\left(\omega_{a}-\omega_{b}\right) t}\right)+\operatorname{Re}\left(\frac{1}{2} a_{1} b_{1} e^{i\left(\omega_{a}+\omega_{b}\right) t}\right)
\end{aligned}
$$

The function comprises a constant term of order 0 , two first-order terms oscillating harmonically with frequencies $\omega_{a}$ and $\omega_{b}$, and two second-order terms oscillating harmonically with frequencies $\omega_{a}-\omega_{b}$ and $\omega_{a}+\omega_{b}$.

Like before, equation (28) is considered as the general form of time dependence occurring in second-order perturbation analyses resulting from interactions of vibrations of two different frequencies

$$
\begin{aligned}
c=c_{0} & +\operatorname{Re}\left(c_{1} e^{i \omega_{1} t}\right)+\operatorname{Re}\left(c_{2} e^{i \omega_{2} t}\right)+\operatorname{Re}\left(c_{3} e^{\left(\omega_{1}-\omega_{2}\right) t}\right) \\
& +\operatorname{Re}\left(c_{4} e^{\left(\omega_{1}+\omega_{2}\right) t}\right)
\end{aligned}
$$

This time function is represented by the second-order two-frequency perturbator

$\mathbf{c}=\left\langle c_{0}, c_{1}, c_{2}, c_{3}, c_{4}\right\rangle$

The constants $c_{1}-c_{4}$ are complex, only $c_{0}$ is real.

One-frequency perturbators are not treated as a special case of two-frequency perturbators having $\omega_{a}=\omega_{b}$. The reasons are as follows:

- Two-frequency perturbators have two first-order elements, while one-frequency perturbators have only one.

- In one-frequency perturbators, the stationary second-order element is, usually, $\neq 0$; it may be of main interest, for example, as the added resistance in waves or as drift force which must be counteracted by dynamic positioning equipment. In twofrequency perturbators, the corresponding element 
represents an oscillation which has an average value of 0 .

- The type (complex or real) of the first second-order quantity is different in one-frequency and twofrequency perturbators.

Both kinds of perturbators may be of interest in different cases. To determine drift forces and moments, that is, the time average of second-order responses, requires one-frequency perturbators, whereas general sum-frequency responses, which are important for ship springing and ringing of tension-leg platforms, require two-frequency perturbators.

Definitions and computing rules for two-frequency perturbators correspond closely to those of onefrequency perturbators. Thus, to change, for example, a program computing drift forces to one that computes difference-frequency responses, is simple: essentially, only the type of the perturbators and the definition of their basic operations have to be changed.

Here, only the most important definition of the general multiplication is given for second-order twofrequency perturbators. It follows from equations (28) and (30)

$$
\mathbf{a} * \mathbf{b}=\left\langle a_{0} * b_{0}, b_{0} * a_{1}, a_{0} * b_{1}, \frac{1}{2} a_{1} * b_{1}^{*}, \frac{1}{2} a_{1} * b_{1}\right\rangle
$$

\section{Second-order pressure force and moment on a cylinder moving in a regular wave}

To demonstrate the application of second-order onefrequency perturbators, the concept is applied here to a 2D test case: a partly submerged cylinder with horizontal axis. The cylinder is moving harmonically in a regular wave. Because the analysis is up to second order, we cannot treat the different degrees of freedom of motions and the wave independently, as is common practice in first-order analyses. Body motion and wave are assumed to have the same frequency, for instance, because the body motions are caused by the wave. The water is presumed to be deep.

By applying Green's integral identities, it is possible to determine the second-order body force from the first-order potential computed for frequencies $\omega$ and $2 \omega$. This was shown, for instance, in Söding. ${ }^{1}$ Here, a more direct method is applied, which is also suitable to determine the second-order pressures: the second-order flow potential is determined using second-order onefrequency perturbators.

\section{Definitions and fundamental relations}

Two Cartesian coordinate systems are used: an inertial system $x^{I}, y^{I}, z^{I}$ and a body-fixed system $x, y, z$ (Figure 1). Both systems coincide in the time average. The origin of the inertial system is in the undisturbed water surface; $x$ points into the direction of the cylinder

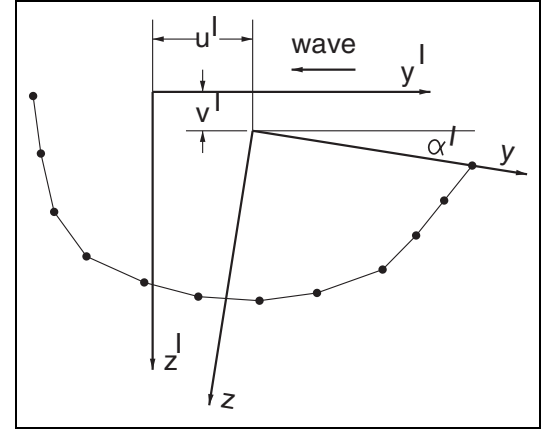

Figure I. Coordinate systems and motion definition.

axis and $z^{I}$ points downward. The wave is running in $-y^{I}$ direction.

The flow is 2D in the $y^{I}, z^{I}$ plane. In spite of this, 3D vectors are used in the following to allow using the vector product and to alleviate extensions to $3 \mathrm{D}$ cases of practical interest. The water is approximated as ideal fluid. The flow is described by a potential $\phi\left(\vec{x}^{I}, t\right)$.

The submerged part of the cylinder's cross section is described by body-fixed offset points starting at the left and ending at the right intersection of the cylinder with the average rest waterline $z=0$. The straight lines between successive offset points are used as body panels.

The cylinder motions are assumed to have 3 degrees of freedom: a horizontal shift (sway) $u^{I}(t)$ of the bodyfixed coordinate origin, a vertical shift (heave) $v^{I}(t)$ of the same point, and a rotation about the $x^{I}$ axis (roll) designated as $\vec{\alpha}^{I}(t)=(\alpha, 0,0)$. Here and in the following, the upper index $I$ indicates that the vector is expressed in the inertial coordinate system.

Compared to its average position, a body-fixed point of position vector $\vec{x}$ changes its inertial coordinates because of the body motions by the vector (correct up to second order)

$$
\vec{\beta}^{I}(\vec{x})=\underbrace{\left(0, u^{I}, v^{I}\right)}_{\vec{\beta}_{0}^{I}}+\vec{\alpha}^{I} \times \vec{x}+\frac{1}{2} \vec{\alpha}^{I} \times\left(\vec{\alpha}^{I} \times \vec{x}\right)
$$

Vectors $\vec{v}$ that, unlike position vectors, do not change with body translations are transformed from the bodyfixed system to the inertial system up to first order according to the equation

$$
\vec{v}^{I}=\vec{v}+\vec{\alpha}^{I} \times \vec{v}
$$

Correspondingly, the vector of space derivatives is transformed

$$
\nabla^{I}=\nabla+\vec{\alpha}^{I} \times \nabla
$$

Using the above relations, $\vec{\beta}^{I}$ can be transformed to the body-fixed coordinate system

$$
\vec{\beta}=\vec{\beta}^{I}-\vec{\alpha}^{I} \times \vec{\beta}^{I}=\vec{\beta}_{0}^{I}+\vec{\alpha}^{I} \times \vec{x}-\vec{\alpha}^{I} \times \vec{\beta}_{0}^{I}-\frac{1}{2} \vec{\alpha}^{I} \times\left(\vec{\alpha}^{I} \times \vec{x}\right)
$$


When computing the flow, it is preferable to deal not with time functions, but with the perturbators representing $\vec{\beta}_{0}^{I}$ and $\vec{\alpha}^{I}$

$$
\overrightarrow{\mathbf{b}}_{0}=\left\langle 0,\left(0, \hat{u}^{I}, \hat{v}^{I}\right), 0,0\right\rangle
$$

and

$$
\overrightarrow{\mathbf{a}}^{I}=\left\langle 0,\left(\hat{\alpha}^{I}, 0,0\right), 0,0\right\rangle
$$

where the hat symbol designates the complex amplitudes of the harmonic motions $u, v, \alpha$. (If the motions contain also constant and/or double-frequency contributions, these can be included in the above definition of the perturbators; the following equations would remain unchanged.)

The perturbator $\overrightarrow{\mathbf{b}}$ of $\vec{\beta}$ follows from equation (35)

$$
\overrightarrow{\mathbf{b}}=\overrightarrow{\mathbf{b}}_{0}+\overrightarrow{\mathbf{a}}^{I} \times \vec{x}-\overrightarrow{\mathbf{a}}^{I} \times \overrightarrow{\mathbf{b}}_{0}-\frac{1}{2} \overrightarrow{\mathbf{a}}^{I} \times\left(\overrightarrow{\mathbf{a}}^{I} \times \vec{x}\right)
$$

The flow is described by a potential which is the sum of the wave potential $\phi_{w}$ and a disturbance (radiation and diffraction) potential $\phi_{d}$

$$
\phi=\phi_{w}+\phi_{d}
$$

The wave potential is the well-known Airy potential

$$
\phi_{w}=-i c \hat{w} e^{-k z^{I}+i k y^{I}} e^{i \omega t}
$$

where $k=\omega^{2} / g$ is the wave number, $c=\omega / k$ is the phase velocity, and $\hat{w}$ is the complex amplitude of the wave. At fixed points (but not on the water surface which involves second-order terms), the Airy potential is correct up to second order; the same holds for the dispersion relation $k=\omega^{2} / g$.

The disturbance potential is approximated as a superposition of the 2D potentials of point sources located at positions $\vec{x}_{j}^{I}$ and having source strengths $q_{j}(t)$

$$
\phi_{d}\left(\vec{x}^{I}\right)=\sum_{j} G\left(\vec{x}^{I}-\vec{x}_{j}^{I}\right) q_{j}(t)
$$

with

$$
G\left(\vec{x}^{I}-\vec{x}_{j}^{I}\right)=\frac{1}{2} \log \left|\vec{x}^{I}-\vec{x}_{j}^{I}\right|^{2}
$$

That ensures satisfaction of the volume conservation law expressed by the Laplace equation $\Delta \phi=0$.

Usual panel methods do not use point sources, but sources distributed over the panels, or over a nearby surface. The use of point sources necessitates to satisfy the boundary conditions not at collocation points, but on average over each panel. This is the $2 \mathrm{D}$ analog of the so-called patch method, ${ }^{2}$ which is held to give more accurate body forces than first-order panel methods for the same discretization. Additionally, it results, usually, in simpler formulae for the influence of sources on the boundary conditions. However, because point sources result in a potential that oscillates around the correct one with wavelengths equal to the panel length, spatial derivatives of the potential cannot be determined (at least not accurately) by the corresponding derivatives of the source potentials. Instead, they are determined as panel averages: tangential derivatives from the difference in the potential at both panel ends and normal derivatives from the flux through the panel. The latter is simply the angle under which the panel is "seen" from the source.

Here, sources (also those above the water surface) are assumed fixed not in the inertial, but in the bodyfixed coordinate system. This has the effect that second derivatives of $\phi$ do not occur in the body boundary condition, but in the free-surface condition. The opposite would be true if sources were fixed in the inertial frame. Because second derivatives can be determined only with relatively large discretization errors, it is better to avoid their occurrence on the body surface where accurate pressure values are required to compute the body force and moment accurately.

The change from inertial to body-fixed coordinates is performed up to second order by using a first-order Taylor expansion of $G$ and applying equation (32)

$$
\begin{aligned}
& \vec{x}^{I}=\vec{x}+\vec{\beta}^{I}(\vec{x}, t) ; \quad \vec{x}_{j}^{I}=\vec{x}_{j}+\vec{\beta}^{I}\left(\vec{x}_{j}, t\right) \\
& \phi\left(\vec{x}^{I}, t\right)=\phi_{w}\left(\vec{x}^{I}, t\right)+\sum_{j} G\left(\vec{x}-\vec{x}_{j}\right) q_{j}(t) \\
& +\sum_{j} \nabla G\left(\vec{x}-\vec{x}_{j}\right) \underbrace{\left[\vec{\beta}^{I}(\vec{x}, t)-\vec{\beta}^{I}\left(\vec{x}_{j}, t\right)\right]}_{\vec{\alpha}^{I} \times\left(\vec{x}-\vec{x}_{j}\right)} q_{j}(t)
\end{aligned}
$$

The last term on the right-hand side is 0 because $\vec{\alpha}^{I} \times\left(\vec{x}-\vec{x}_{j}\right)$ is a vector being perpendicular to $\vec{x}-\vec{x}_{j}$, while $\nabla G\left(\vec{x}-\vec{x}_{j}\right)$ is a vector in the direction of $\vec{x}-\vec{x}_{j}$. Thus

$$
\phi\left(\vec{x}^{I}, t\right)=\phi_{w}\left(\vec{x}^{I}, t\right)+\sum_{j} G\left(\vec{x}-\vec{x}_{j}\right) q_{j}(t)
$$

The occurrence of $\dot{\phi}$ in Bernoulli's equation requires to distinguish between the function giving the flow potential depending on $\vec{x}^{I}$, from that giving it as a function of $\vec{x}$

$$
\phi\left(\vec{x}^{I}, t\right)=\Phi(\vec{x}, t)
$$

if $\vec{x}^{I}$ and $\vec{x}$ designate the same point, that is, if $\vec{x}^{I}=\vec{x}+\vec{\beta}$.

Taking the partial time derivative for constant $\vec{x}^{I}$, which is required in Bernoulli's equation, gives

$$
\dot{\phi}\left(\vec{x}^{I}, t\right)=\dot{\Phi}(\vec{x}, t)+\nabla \Phi \dot{\vec{x}}
$$

where $\dot{\vec{x}}$ is meant to hold for fixed $\vec{x}^{I}$. Because $\vec{x}^{I}=\vec{x}+\vec{\beta}$, we have $\dot{\vec{x}}=-\vec{\beta}$ and thus

$$
\dot{\phi}\left(\vec{x}^{I}, t\right)=\dot{\Phi}(\vec{x}, t)-\nabla \Phi \overrightarrow{\vec{\beta}}
$$

and

$$
\ddot{\phi}=\ddot{\Phi}-2 \nabla \dot{\Phi} \dot{\vec{\beta}}-\nabla \Phi \ddot{\vec{\beta}}
$$


From equation (34) follows the relation between the gradients of $\phi$ and $\Phi$

$$
\nabla^{I} \phi=\nabla \Phi+\vec{\alpha}^{I} \times \nabla \Phi
$$

In the following, the perturbator $\boldsymbol{\Phi}(\vec{x})$ will be used to represent the time function $\Phi(\vec{x}, t)$.

\section{Body boundary condition}

The body boundary condition states that there is no flux through the cross section contour

$$
\nabla^{I} \phi\left(\vec{x}^{I}, t\right) \vec{n}^{I}=\dot{\vec{\beta}}^{I}(\vec{x}) \vec{n}^{I}
$$

The condition is to be satisfied on the submerged part of the cross section. $\vec{n}^{I}$ is the normal vector (pointing into the body) on the section contour, expressed in inertial coordinates.

Using equations (34) and (45), the left-hand side of equation (51) can be transformed to

$$
\begin{aligned}
& \nabla^{I} \phi\left(\vec{x}^{I}, t\right) \vec{n}^{I}=\nabla^{I} \phi_{w}\left(\vec{x}^{I}, t\right) \vec{n}^{I} \\
& +\sum_{j} \nabla G\left(\vec{x}-\vec{x}_{j}\right) q_{j}\left(\vec{n}+\vec{\alpha}^{I} \times \vec{n}\right)+\vec{\alpha}^{I} \\
& \times \sum_{j} \nabla G\left(\vec{x}-\vec{x}_{j}\right) q_{j} \vec{n} \\
& =\nabla^{I} \phi_{w}\left(\vec{x}^{I}, t\right) \vec{n}^{I}+\sum_{j} \nabla G\left(\vec{x}-\vec{x}_{j}\right) q_{j} \vec{n}
\end{aligned}
$$

The term involving the wave potential $\phi_{w}$ must also be changed to time-invariable quantities. Using equation (40), one finds

$$
\nabla^{I} \phi_{w}\left(\vec{x}^{I}, t\right) \vec{n}^{I}=-i \omega \hat{w} e^{i \omega t} e^{k \vec{w} \vec{x}} \vec{w}\left[\vec{n}(1+k \vec{w} \vec{\beta})+\vec{\alpha}^{I} \times \vec{n}\right]
$$

where

$$
\vec{w}=(0, i,-1)
$$

The right-hand side of equation (51) is transformed to the body-fixed coordinate system using equation (33)

$$
\begin{aligned}
\dot{\vec{\beta}}^{I} \vec{n}^{I}= & \frac{\partial}{\partial t}\left(\vec{\beta}^{I} \vec{n}^{I}\right)-\vec{\beta}^{I} \dot{\vec{n}}^{I}=\frac{\partial}{\partial t}(\vec{\beta} \vec{n}) \\
& -\left(\dot{\vec{\alpha}}^{I} \times \vec{n}\right) \vec{\beta}=\dot{\vec{\beta}} \vec{n}-\left(\dot{\vec{\alpha}}^{I} \times \vec{n}\right) \vec{\beta}
\end{aligned}
$$

Thus, the body boundary condition is

$$
\sum_{j} \nabla G\left(\vec{x}-\vec{x}_{j}\right) q_{j} \vec{n}=-\nabla^{I} \phi_{w}\left(\vec{x}^{I}, t\right) \vec{n}^{I}+\dot{\vec{\beta}} \vec{n}-\left(\dot{\vec{\alpha}}^{I} \times \vec{n}\right) \vec{\beta}
$$

Using equation (53), the perturbator form of the condition becomes

$$
\begin{gathered}
\sum_{j} \nabla G\left(\vec{x}-\vec{x}_{j}\right) \vec{n} \mathbf{q}_{j}=\left\langle 0, i \omega \hat{w} e^{k \vec{w} \vec{x}} \vec{w}, 0,0\right\rangle \\
{\left[\vec{n}+k \vec{n}(\vec{w} \overrightarrow{\mathbf{b}})+\overrightarrow{\mathbf{a}}^{I} \times \vec{n}\right]+\dot{\overrightarrow{\mathbf{b}}} \vec{n}-\left(\dot{\overrightarrow{\mathbf{a}}}^{I} \times \vec{n}\right) \overrightarrow{\mathbf{b}}}
\end{gathered}
$$

\section{Boundary condition at the water surface}

While sources and body panels are fixed in the body coordinate system, that is, they follow the body motions, panels on the water surface are fixed in the inertial system, that is, they are arranged on the line $z^{I}=0$ at both sides of the body up to a reasonable distance from it.

The vertical coordinate $z^{I}$ of the free surface is designated as $\zeta\left(y^{I}, t\right)$. Bernoulli's equation, and the fact that there is atmospheric pressure at the free surface, results in the free-surface boundary condition

$$
g \zeta-\dot{\phi}-\frac{1}{2}(\nabla \phi)^{2}=0
$$

which is to be satisfied at $z^{I}=\zeta\left(y^{I}\right)$ outside of the body. By means of a Taylor expansion, the condition is converted (up to second order) to a condition to be satisfied on the plane $z^{I}=0$

$$
\begin{aligned}
g \zeta-\dot{\phi}-\zeta \dot{\phi}_{z}-\frac{1}{2}(\nabla \phi)^{2}=g \zeta-\dot{\phi}-\frac{1}{g} \dot{\phi}_{\dot{\phi}} \\
-\frac{1}{2}(\nabla \phi)^{2}=0
\end{aligned}
$$

where index $z$ designates a partial derivative with respect to $z^{I}$. The second expression in equation (59) was derived by eliminating $\zeta$ in the third term, using a first-order approximation to $\zeta$ following from equation (58). The above equation can be solved for $\zeta$ as a function of $\phi$ derivatives

$$
\zeta=\frac{1}{g}\left(\dot{\phi}+\frac{1}{g} \dot{\phi} \dot{\phi}_{z}+\frac{1}{2}(\nabla \phi)^{2}\right)
$$

where all $\phi$ derivatives must be evaluated on $z^{I}=0$.

There is also no flux through the water surface. This is expressed by

$$
\frac{D}{D t}\left(\zeta-z^{I}\right)=\left(\frac{\partial}{\partial t}+\nabla^{I} \phi \nabla^{I}\right)\left(\zeta-z^{I}\right)=0
$$

where $D / D t$ indicates the substantial time derivative. Evaluating the partial derivatives of $z^{I}$ and $\zeta$ gives

$$
\dot{\zeta}+\phi_{y} \zeta_{y}-\phi_{z}=0
$$

on the free surface $z^{I}=\zeta$. Again, the equation is transformed to $z^{I}=0$ using a Taylor expansion

$$
\dot{\zeta}+\phi_{y} \zeta_{y}-\phi_{z}-\zeta \phi_{z z}=0
$$

Eliminating $\zeta$ by using equation (60), substituting $\phi_{z z}$ by $-\phi_{y y}$ because of the continuity equation, and rearranging terms give 


$$
-\ddot{\phi}+g \phi_{z}=\frac{1}{g} \frac{\partial}{\partial t}\left(\dot{\phi} \dot{\phi}_{z}\right)+\nabla \phi \nabla \dot{\phi}+\frac{\partial}{\partial y}\left(\dot{\phi} \phi_{y}\right)
$$

For the second-order terms on the right-hand side, it is arbitrary whether they are evaluated at $\vec{x}^{I}$ or at $\vec{x}$ because this difference is of third order. For the first-order terms on the left-hand side, however, this difference is important. The free-surface panels have fixed $\vec{x}^{I}$. Thus, we have to insert $\vec{x}^{I}-\vec{\beta}$ for $\vec{x}$ in equation (45)

$$
\begin{gathered}
\phi\left(\vec{x}^{I}, t\right)=\phi_{w}+\phi_{d}=\phi_{w}\left(\vec{x}^{I}, t\right)+\sum_{j} G\left(\vec{x}^{I}-\vec{\beta}-\vec{x}_{j}\right) q_{j}(t) \\
=\phi_{w}\left(\vec{x}^{I}, t\right)+\underbrace{\sum_{j} G\left(\vec{x}^{I}-\vec{x}_{j}\right) q_{j}(t)}_{\Phi_{d}\left(\vec{x}^{I}\right)}-\vec{\beta} \nabla \Phi_{d}
\end{gathered}
$$

The left-hand side of equation (64) is therefore

$$
\begin{aligned}
& -\ddot{\phi}+g \phi_{z}=-\ddot{\phi}_{w}+g \phi_{w z} \\
& +\sum_{j}\left[-G\left(\vec{x}^{I}-\vec{x}_{j}\right) \ddot{q}_{j}+g G_{z}\left(\vec{x}^{I}-\vec{x}_{J}\right) q_{j}\right] \\
& +g \alpha^{I} \phi_{d y}+\frac{\partial^{2}}{\partial t^{2}}\left(\vec{\beta} \nabla \phi_{d}\right)-g \vec{\beta} \nabla \phi_{d z}
\end{aligned}
$$

The expression $-\ddot{\phi}_{w}+g \phi_{w z}$ is 0 . Thus, the freesurface boundary condition becomes

$$
\begin{aligned}
& \sum_{j}\left[-G\left(\vec{x}^{I}-\vec{x}_{j}\right) \ddot{q}_{j}+g G_{z}\left(\vec{x}^{I}-\vec{x}_{J}\right) q_{j}\right] \\
& =-g \alpha^{I} \phi_{d y}-\frac{\partial^{2}}{\partial t^{2}}\left(\vec{\beta} \nabla \phi_{d}\right)+g \vec{\beta} \nabla \phi_{d z}+\frac{1}{g} \frac{\partial}{\partial t}\left(\dot{\phi} \dot{\phi}_{z}\right) \\
& +\nabla \phi \nabla \dot{\phi}+\frac{\partial}{\partial y}\left(\dot{\phi} \phi_{y}\right)
\end{aligned}
$$

The corresponding perturbator equation is

$$
\begin{aligned}
& \sum_{j}\left[-G\left(\vec{x}^{I}-\vec{x}_{j}\right) \ddot{\mathbf{q}}_{j}+g G_{z}\left(\vec{x}^{I}-\vec{x}_{J}\right) \mathbf{q}_{j}\right] \\
& =-g \mathbf{a}^{I} \boldsymbol{\Phi}_{d y}-\left(\overrightarrow{\mathbf{b}} \nabla \boldsymbol{\Phi}_{d}\right)^{\bullet \bullet}+g \overrightarrow{\mathbf{b}} \nabla \boldsymbol{\Phi}_{d z}+\frac{1}{g}\left(\dot{\boldsymbol{\Phi}} \dot{\boldsymbol{\Phi}}_{z}\right)^{\bullet} \\
& +\nabla \boldsymbol{\Phi} \nabla \dot{\boldsymbol{\Phi}}+\frac{\partial}{\partial y}\left(\dot{\boldsymbol{\Phi}} \boldsymbol{\Phi}_{y}\right)
\end{aligned}
$$

\section{Further boundary conditions}

The velocity $\nabla \phi$ and the pressure oscillations, whichto first order - are proportional to $\phi$, vanish for $z^{I} \rightarrow \infty$ (in great depth). That condition could be satisfied for the disturbance potential by requiring that the sum of source strengths is 0 . However, it turned out that this condition is not necessary here and that it did not improve the consistency of the numerical results. Therefore, no such condition is applied.

We want to compute the flow generated alone by the incoming wave and the (moving or stationary) body, not by other bodies, wave reflections at walls, and so on. This is usually expressed by admitting only disturbance waves which, for $y^{I} \rightarrow+\infty$ and $y^{I} \rightarrow-\infty$, run in $+y^{I}$ or $-y^{I}$ direction, respectively. This socalled radiation condition is satisfied here by damping the waves. This is most easily effected by applying in the free-surface condition a complex gravity acceleration $g$. Its real part has the correct physical value, the imaginary part (it must be positive) is assumed 0 at both body sides, and increasing quadratically with distance from the body. Thus, waves near the body are only slightly affected, but farther outside the waves are damped heavily. This avoids not only reflections at the end of the discretized range of the free surface; it helps also to avoid that body forces and other results depend on whether the discretized free-surface length contains an even or odd number of half wavelengths.

\section{Details of the discretization}

The body contour is approximated by straight "panels" between successive offset points. For each panel, the flux due to the wave and all source potentials is set to 0 . On the mean free surface $z_{I}=0, n_{f}$ panels are generated on either side of the body. For each of them, condition (69), integrated over the panel length, is satisfied; $n_{f}=40$ is more than enough to compute accurate results for first-order force and moment. However, second-order forces are much more sensitive. To avoid unnecessary complexity, first- and second-order source strengths are determined using the same panel arrangement with $n_{f}=70$. The length of free-surface panels adjacent to the body is chosen equal to the length of the uppermost body panels. The next outer panels increase in length by a constant factor up to a maximum length of $1 / 15$ of the wavelength.

The integrals over panel length of the wave potential and its derivatives are evaluated analytically, while the right-hand side of equation (69) is evaluated numerically using five points per panel. Other methods proved to work even well.

Sources are arranged within the body, typically $75 \%$ of the panel length shifted inside in normal directions from each panel midpoint. Sources above the freesurface panels are arranged correspondingly above the water surface.

\section{Determination of source strengths}

The body boundary condition (57) is integrated separately over each body panel; this gives conditions for the source strength perturbators $q_{j}$. Further conditions result from integrating the free-surface boundary condition (69) separately over each free-surface panel. The number of conditions is equal to the number of sources. The source strength perturbators appear only linearly in these conditions. The matrix of this linear equation system is not of perturbator type, but complex because of the complex $g$; otherwise it would be real. Only the right-hand sides and the unknowns are perturbators. 
To determine the source strength perturbators, it appears best not to generate a linear equation solver for perturbator-type variables, but to use a solver for complex linear equations, and to apply it by splitting the unknown source perturbators and the right-hand side perturbators into their respective perturbator elements. All zero-order elements are 0 here. Thus, we obtain three equation systems for the perturbator elements 1,2 , and 3 .

In detail, equations (57) and (69) are combined and written formally as an equation system

$$
\sum_{j}\left(\bar{K}_{i j} \ddot{\mathbf{q}}_{j}+K_{i j} \mathbf{q}_{j}\right)=\mathbf{R}_{i}
$$

$\bar{K}_{i j}$ and $K_{i j}$ are quadratical complex matrices. $\bar{K}_{i j}$ is $\neq 0$ only in the lines corresponding to free-surface panels.

The perturbators in this equation are split into their elements

$$
\begin{aligned}
\ddot{\mathbf{q}}_{j} & =\left\langle 0,-\omega^{2} q_{j}^{(1)}, 0,-4 \omega^{2} q_{j}^{(3)}\right\rangle \\
\mathbf{q}_{j} & =\left\langle 0, q_{j}^{(1)}, q_{j}^{(2)}, q_{j}^{(3)}\right\rangle \\
\mathbf{R}_{i} & =\left\langle 0, R_{i}^{(1)}, R_{i}^{(2)}, R_{i}^{(3)}\right\rangle
\end{aligned}
$$

Here, perturbator elements are designated by upper indices in parentheses. $q_{j}^{(1)}$ and $q_{j}^{(3)}$ are complex, and $q_{j}^{(2)}$ are real.

Applying the rule for multiplying perturbators by complex constants (here $\bar{K}_{i j}$ and $K_{i j}$ ) gives three linear equation systems

$$
\begin{aligned}
& \sum_{j}\left(-\omega^{2} \bar{K}_{i j}+K_{i j}\right) q_{j}^{(1)}=R_{i}^{(1)} \\
& \sum_{j} \operatorname{Re}\left(K_{i j}\right) q_{j}^{(2)}=R_{i}^{(2)} \\
& \sum_{j}(\operatorname{Re}+2 i \operatorname{Im})\left(-4 \omega^{2} \bar{K}_{i j}+K_{i j}\right) q_{j}^{(3)}=R_{i}^{(3)}
\end{aligned}
$$

The variables appearing in systems 1 and 3 are complex; in system 2, they are real.

System (74) must be solved first because the freesurface elements of $R_{i}^{(2)}$ and $R_{i}^{(3)}$ contain the initially unknown perturbators of the potential $\boldsymbol{\Phi}$ (as shown in equation (69)); the corresponding parts of $R_{i}^{(1)}$ are 0 because the right-hand side of equation (69) is of purely second-order (its first-order part is 0 ).

System (75) gives the stationary second-order source strengths. These do not contribute to the body pressure and force up to second order; thus, they need not be determined except if the stationary second-order flow is of interest.

When system (74) has been solved, the first-order potential is computed and used to determine the righthand sides of system (76); no higher order contributions are required to determine the right-hand sides of equations (57) and (69). When system (76) is solved for $q_{j}^{(3)}$, the perturbators of source strengths

$$
\mathbf{q}_{j}=\left\langle 0, q_{j}^{(1)}, 0, q_{j}^{(3)}\right\rangle
$$

are known.

\section{Determination of second-order pressure}

The source perturbators $\mathbf{q}_{j}$ allow to determine the perturbators of the potential at all body offset points by using the perturbator equivalence to equation (45)

$$
\boldsymbol{\Phi}(\vec{x})=\left\langle 0,-i c \hat{w} e^{k \vec{w} \vec{x}}, 0,0\right\rangle(1+k \vec{w} \overrightarrow{\mathbf{b}})+\sum_{j} G\left(\vec{x}-\vec{x}_{j}\right) \mathbf{q}_{j}
$$

To determine the pressure force, we need the fluid pressure (minus atmospheric pressure). It follows from Bernoulli's equation

$$
p(\vec{x}, t)=\rho\left(g z^{I}-\dot{\phi}\left(\vec{x}^{I}, t\right)-\frac{1}{2}(\nabla \phi)^{2}\right)
$$

Using equation (48) gives

$$
p(\vec{x}, t)=\rho\left(g(\vec{x}+\vec{\beta})_{3}-\dot{\Phi}(\vec{x}, t)+\nabla \Phi \dot{\vec{\beta}}-\frac{1}{2}(\nabla \Phi)^{2}\right)
$$

where index 3 designates the $z$ component. The corresponding perturbator equation is

$$
\mathbf{p}(\vec{x})=\rho\left(g(\vec{x}+\overrightarrow{\mathbf{b}})_{3}-\dot{\boldsymbol{\Phi}}(\vec{x})+\nabla \boldsymbol{\Phi} \dot{\vec{b}}-\frac{1}{2}(\nabla \boldsymbol{\Phi})^{2}\right)
$$

\section{Determination of body force and moment}

The body force and moment per length result from two contributions: the pressure integrated along the section contour up to the average immersion and the effect of time-varying immersion. The perturbators of the first contribution are

$$
\overrightarrow{\mathbf{F}}_{1}=\sum_{k} \mathbf{p}_{k} \vec{f}_{k}
$$

for the force vector and

$$
\overrightarrow{\mathbf{M}}_{1}=\sum_{k} \mathbf{p}_{k}\left(\vec{x}_{k} \times \vec{f}_{k}\right)
$$

for the moment. Here, $\vec{f}_{k}$ is the panel length vector: normal on the panel, pointing inward, length $=$ panel length; and $\vec{x}_{k}$ is the position vector of the panel midpoint. Only the $x$ component of $\overrightarrow{\mathbf{M}}_{1}$ is nonzero. Both force and moment are expressed in the body-fixed coordinate system; the moment refers to the body-fixed origin.

The contribution due to time-varying immersion depends on the pressure $p_{i}$ at the points of intersection between section contour and average waterline $z=0$; $i=1$ designates the left and $i=2$ the right intersection. The difference between actual and time-averaged 
$z$-coordinate of the free surface is (up to first order, which is sufficient here)

$$
z_{i}=\frac{-p_{i}}{\rho g}
$$

If $p_{i}>0$, the wetted body contour length is larger than its time average value by

$$
\frac{-z_{i}}{\left|n_{2 i}\right|}=\frac{p_{i}}{\rho g\left|n_{2 i}\right|}
$$

where $n_{2 i}$ is the $y$ component of the unit normal vector at point $i$. The increased immersion causes an additional force

$$
\frac{1}{2} p_{i} \cdot \frac{p_{i}}{\rho g\left|n_{2 i}\right|} \vec{n}_{i}
$$

The first factor gives the spatially averaged pressure between actual waterline (where $p=0$ ) and average waterline at $z=0$. In case of $p_{i}<0$, the pressure integration up to $z=0$ contains a contribution from a part of the body contour which is actually emersed; the spatially averaged pressure there is the negative value $1 / 2 p_{i}$. Thus, also in this case, a correction of size (86) must be added. The perturbators of the second force and moment contribution, which results from this correction on both sides of the body, are therefore

$$
\begin{aligned}
& \overrightarrow{\mathbf{F}}_{2}=\sum_{i=1}^{2} \frac{1}{2 \rho g\left|n_{2 i}\right|} \mathbf{p}_{i}^{2} \vec{n}_{i} \\
& \overrightarrow{\mathbf{M}}_{2}=\sum_{i=1}^{2} \frac{1}{2 \rho g\left|n_{2 i}\right|} \mathbf{p}_{i}^{2} \vec{x}_{i} \times \vec{n}_{i}
\end{aligned}
$$

In evaluating these formulae, $\mathbf{p}_{i}$ is extrapolated to $z=0$ from the average values within the uppermost two body panels on either side.

After adding both contributions, the force and moment perturbators are changed to the inertial coordinate system

$$
\overrightarrow{\mathbf{F}}^{I}=\overrightarrow{\mathbf{F}}+\overrightarrow{\mathbf{a}}^{I} \times \overrightarrow{\mathbf{F}} ; \quad \overrightarrow{\mathbf{M}}^{I}=\overrightarrow{\mathbf{M}}+\overrightarrow{\mathbf{a}}^{I} \times \overrightarrow{\mathbf{M}}
$$

Note that the reference point for the moment is still the body-fixed coordinate origin.

\section{Results}

For a semi-submerged circular cylinder in sinusoidal heave motion, the vertical force per length perturbator $\overrightarrow{\mathbf{F}}_{z}$ is determined depending on the heave frequency. (The horizontal force and the roll moment are 0 in this case.) The elements of the vector force perturbator are designated as follows

$$
\overrightarrow{\mathbf{F}}_{z}=\left\langle F_{z}^{(0)}, F_{z}^{(1)}, F_{z}^{(2)}, F_{z}^{(3)}\right\rangle
$$

The stationary buoyancy $F_{z}^{(0)}$ is of no interest here. The three other elements (i.e. the first-order force amplitude, the stationary second-order force, and the

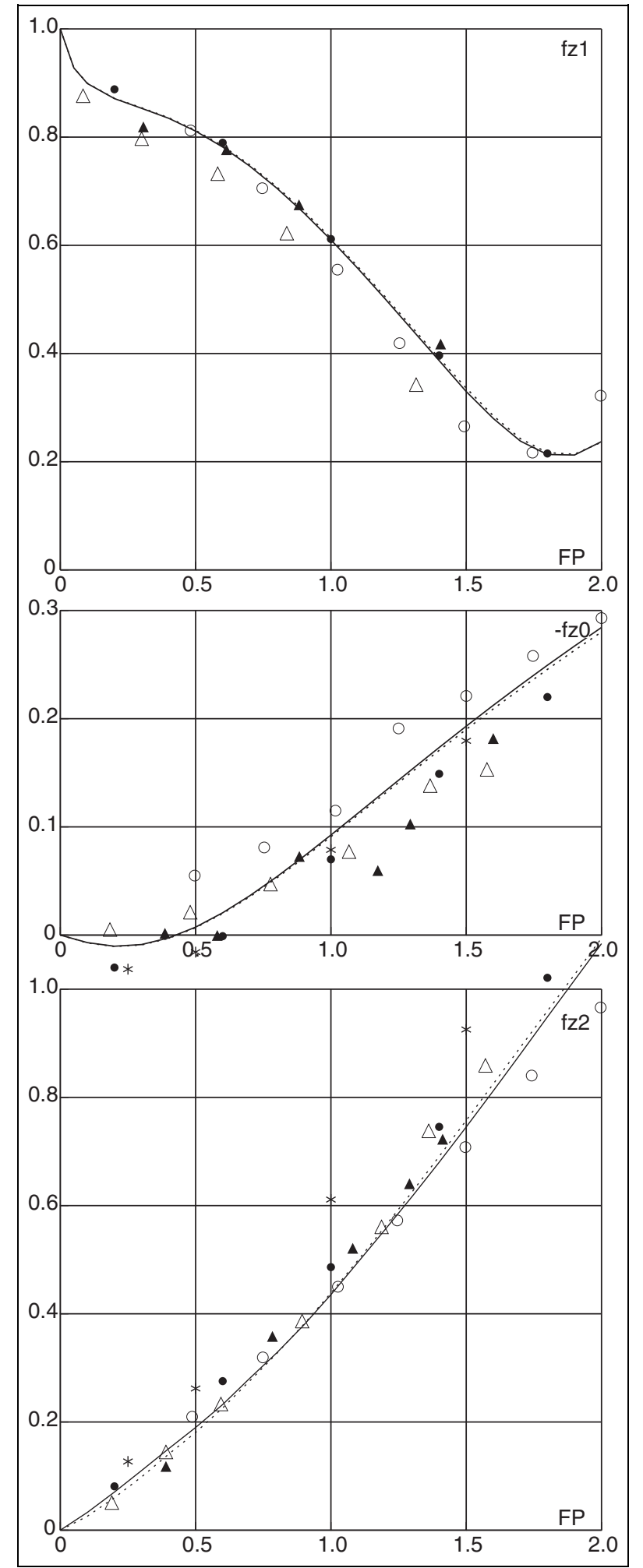

Figure 2. Heaving motion of a semicircle. Curves: present method for 99 (continuous) and 20 (dotted) body panels. Markers indicate published results: dots from Papanikolaou; ${ }^{3}$ asterisks from Söding' (both by potential methods); circles from Gentaz et al. ${ }^{4}$ (Navier-Stokes); and triangles from Yamashita ${ }^{5}$ (second order) and Tasai and Koterayama ${ }^{6}$ (first order) (experiments with $A / R=0.2$ (black) and $A / R=0.4$ (light)).

double-frequency second-order force amplitude) are made nondimensional in the following way 


$$
f_{z 1}=\frac{\left|F_{z}^{(1)}\right|}{2 \rho g A R} ; \quad f_{z 0}=\frac{F_{z}^{(2)}}{2 \rho g A^{2}} ; \quad f_{z 2}=\frac{\left|F_{z}^{(3)}\right|}{2 \rho g A^{2}}
$$

Here, $R$ is the radius of the cylinder and $A$ is the motion amplitude.

Figure 2 shows these nondimensional quantities depending on the frequency parameter

$$
F P=\omega^{2} \frac{R}{g}
$$

The results of this method are compared with published results. The approximate coincidence of the dotted and the continuous curves shows that both for first- and second-order quantities, a discretization with 20 body panels is fully sufficient. (For wave excitation forces in short waves, on the other hand, more than 20 body panels are required for accurate results.) The linear response is in perfect coincidence with the potential calculation, ${ }^{3}$ whereas experimental results and results of Navier-Stokes calculations show reasonable deviations.

The second-order results from different sources show, however, much more scatter. This is to be expected because these responses are usually much smaller than the first-order ones, and they are much more sensitive to discretization and measuring errors. Nonetheless, the current results are well within the range of scatter of the various other published results.

Furthermore, it was found (not demonstrated here) that the results of this method are largely unaffected by reasonable changes in the number of body and freesurface panels, the source locations, the length ratios of adjacent free-surface panels, and the ratio between maximum panel length and wavelength. In Söding, ${ }^{7}$ it is further shown that similar conclusions hold for a fixed cylinder in waves. The method is suitable also for combinations of various kinds of motions and wave excitation, which - unlike first-order responses - cannot be determined by superposition of separate responses.

\section{Conclusion}

Second-order potential flow computations require tedious developments of the boundary conditions; they involve vectors expressed in different coordinate systems and distinguish between functions depending on these different vector representations. All this is not simplified by perturbators. However, the numerical evaluation of the boundary conditions and all further relations for pressures, force, and moment are simplified very much by using perturbators because they eliminate largely the need for subdividing time-dependent quantities into parts of different order and oscillation frequency. Relations that are expressed simply as a product of two perturbators must be described, if perturbators are not used, by expressions corresponding in number and complexity to equation (15).

Computing second-order flows due to superimposing motions and waves of two different frequencies is practically important, for example, in ship springing and platform ringing. When using perturbators, this problem is hardly more complicated than the secondorder single-frequency case which was dealt with here.

Perturbators do not change discretization errors, and they hardly influence computing time: They may increase computing time a little because, when computing with perturbators some elements of which are 0 , unnecessary operations with 0 elements will probably be performed. Much more important appears, however, the simplification of programming when using perturbators as program variables and applying their basic mathematical operations.

The 2D flow analysis described here will serve as a model for extensions to three space dimensions.

\section{Declaration of conflicting interests}

The author(s) declared no potential conflicts of interest with respect to the research, authorship, and/or publication of this article.

\section{Funding}

This research received no specific grant from any funding agency in the public, commercial, or not-for-profit sectors.

\section{References}

1. Söding H. Second-order forces on oscillating cylinders in waves. Schiffstechnik J 1976; 23: 205-209.

2. Söding H. A method for accurate force calculations in potential flow. Ship Techn Res J 1993; 40: 176-186.

3. Papanikolaou A. On calculations of nonlinear hydrodynamic effects in ship motion. Schiffstechnik $J$ 1984; 31: 91129.

4. Gentaz L, Alessandrini B and Delhommeau G. Motion simulation of a cylinder at the surface of a viscous fluid. Ship Techn Res J 1996; 43: 3-18.

5. Yamashita S. Calculations of the hydrodynamic forces acting upon thin cylinders oscillating vertically with large amplitude. J SNAJ 1977; 141: 61-69.

6. Tasai $\mathrm{F}$ and Koterayama W. Nonlinear hydrodynamic forces acting on cylinders heaving on the surface of a fluid. Rep Res Inst Appl Mech Kyushu Univ 1976; 23: 77.

7. Söding H. Second-order seakeeping analyses using perturbators. Ship Techn Res J 2014; 61: 4-15. 